\title{
Monodisperse water microdroplets generated by electrohydrodynamic atomization in the simple-jet mode
}

\author{
L. L. F. Agostinho, ${ }^{1,2}$ C. U. Yurteri, ${ }^{1}$ E. C. Fuchs ${ }^{2}$ and J. C. M. Marijnissen ${ }^{1,2}$ \\ ${ }^{1}$ Faculty of Applied Sciences, Delft University of Technology, Delft 2628 BL, The Netherlands \\ ${ }^{2}$ Wetsus Centre of Excellence for Sustainable Water Technology, Leeuwarden 8900 CC, The Netherlands
}

(Received 29 March 2012; accepted 21 May 2012; published online 12 June 2012)

\begin{abstract}
Experiments were conducted in order to investigate the influences of flow rate, applied voltage, and electric conductivity on droplet size and size distribution of water electrosprays in the simple-jet mode. The results show that the electric potential decreases significantly the relative standard deviation (RSD) of the spray size distribution, with the best result obtained for Weber number, $\mathrm{We}=3.3(240 \mathrm{ml} / \mathrm{h})$ when the RSD decreases from 0.50 at $0 \mathrm{kV}$ to 0.18 at $5 \mathrm{kV}$. We conclude that simple-jet mode electrosprays are a good option for applications which require monodisperse micrometer droplets with high throughput. (C) 2012 American Institute of Physics. [http://dx.doi.org/10.1063/1.4729021]
\end{abstract}

The generation of monodisperse sprays is crucial for many industrial and medical applications. ${ }^{1-7}$ Among others, such sprays can be used to control droplet deposition in inkjet printing, ${ }^{8}$ to improve lung targeting in drug inhalation technology 9,10 and they are known to enhance the evaporation rate in combustion systems. ${ }^{11}$

Commonly, the droplets generated in these processes are formed from the breakup of a liquid ligament. The process is named after the mechanism used to create the filament, e.g., pressure gradients (pressure atomizers), gas streams (air assisted atomizers), centrifugal forces (rotary atomizers), and electrostatic forces (electrohydrodynamic atomizers). 1,12,13 Empirical and theoretical investigations have been done to understand the mechanism responsible for the formation of such sprays. Among them, the most famous one is the study done by Rayleigh ${ }^{14}$ who found that noncompressible inviscid liquid jets are unstable regarding axisymmetric disturbances of wave number $(k)$ less than a certain cut-off wave number, i.e., the critical wave number $\left(k_{c}\right)$ and calculated that the diameter of the formed droplet $(d)$ is related to the jet diameter $(D)$ as $d \approx 1.89 \times D$. From nonlinear theory, we know nowadays that for each disturbance forming a main droplet one or more usually smaller droplets (satellite or secondary droplets) can be formed. ${ }^{1}$ However, it is possible to disturb the jet such that these satellite droplets are not formed, thereby generating a monodisperse spray. ${ }^{1,15}$

From all the above mentioned atomization methods, electrohydrodynamic atomization (EHDA) is one of the few which is capable to generate monodisperse micrometer size droplets. Another unique feature of this method is the electric charge acquired by the droplets which provides self dispersion and prevents coalescence. ${ }^{3,16}$ In view of these appealing characteristics, the production of monodisperse sprays using EHDA has attracted considerable attention in the literature. ${ }^{2-7,10,12,17,18}$ Some examples are the works of Tang and Gomez (1994), who investigated monodisperse electrosprayed water droplets for targeted drug delivery ${ }^{7}$ and monodisperse sprays of low conductivity liquids ${ }^{3}$ as well as the works of Deng et al. (2009) and Arnanthigo et al. (2011) who have developed multiplex systems for the production of such sprays. ${ }^{6,19}$ These papers consider electrospray in the cone-jet mode largely due to the fact that droplets generated in this mode are monodisperse and much smaller than the nozzle diameter; thus, allowing the usage of big nozzles. However, such a mode operates at very low flow rates ${ }^{3,20-22}$ which is a limitation for applications requiring high throughput, ${ }^{19}$ e.g., cooling towers, thermal desalination, and spray drying. A solution for this problem is to operate at higher flow rates, therefore, in a different mode. According to the classifications presented by Cloupeau and Prunet Foch ${ }^{23}$ and Grace and Marijnissen, ${ }^{24}$ one option would be the simple-jet mode.

In the present work we studied the production of monodisperse aqueous electrosprays in the simple-jet mode. The effects of flow rate, applied voltage, and electric conductivity on monodispersity and droplet size were investigated. The liquids used were deionized water and a solution of sodium chloride in deionized water. The focus is on applications which require monodisperse sprays with high throughput and droplets in the hundreds of micrometers size.

To conduct the investigations, electrospray was studied using a nozzle to ring configuration (Figure 1). A blunt ended, polished, stainless steel needle (FED Inc.) was used as nozzle (gauge number 22, $210 \mu \mathrm{m}$ ID and $510 \mu \mathrm{m}$ OD, uncoated). A pump type SIMDOS $^{\circledR}$ Model: FEM 1.10 KT.18S was used to create a constant flow rate (Q) through the nozzle. The liquids used in the experiments were deionized water $(\sim 18 \mathrm{M} \Omega \cdot \mathrm{cm}$, Millipore system), and a solution of deionized $\mathrm{NaCl}$ (99\% Sigma Tech) in deionized water with a concentration of $35 \mathrm{~g} / \mathrm{l}$, from now on referred to as Dwater and $\mathrm{NaCl}_{\mathrm{aq}}$ respectively. Viscosity, density, relative permittivity, conductivity, and surface tension (liquid-air interface) of the liquids are given in Table I.

High voltage was applied with a FUG HCP 35-35 000 DC high voltage power supply. In all experiments, the counter electrode was set on a negative potential $(\Phi)$ whereas the nozzle was grounded. The distance between nozzle and ring was kept constant with the ring placed $1.7 \mathrm{~cm}$ above the nozzle tip as done by Geerse. ${ }^{25}$ A multi-meter (Fluke 8846 A 6.5 digit precision multimeter) connected in series with the 


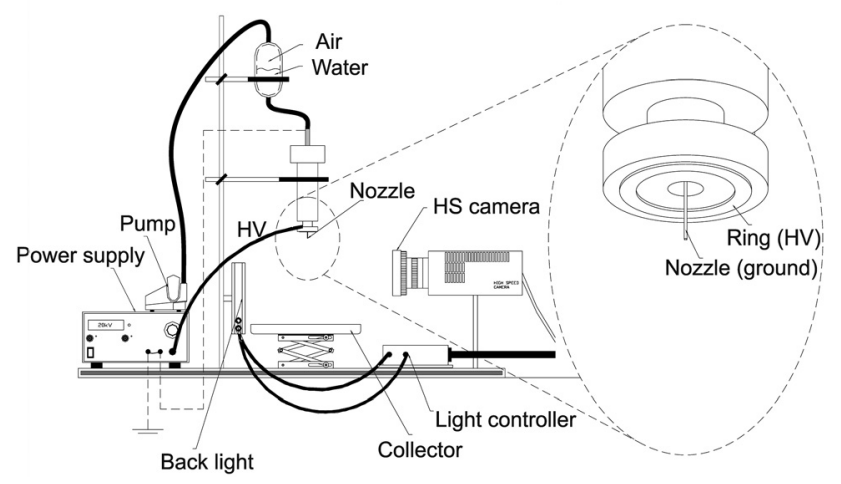

FIG. 1. Electrospray and optical system scheme.

nozzle and the ground (not shown in the picture) was used to measure the electric current through the liquid jet. An optical system consisting of a Photron SA-1 high speed camera (HS camera) and diffused backlight illumination (microscope light source Karl Storz Technolight 270 with liquid light guide and diffuser) was employed to record the experiments. Image $\mathrm{J}^{\circledR}$ was used for image processing; brightness and contrast corrections were done using Corel Photopaint ${ }^{\circledR}$ 15. After being processed, the image data were analyzed statistically using a self made MATLAB ${ }^{\circledR}$ routine.

The liquid Weber number $W e=\frac{\rho_{l} \times r \times v^{2}}{\gamma}$ (where $\rho_{1}$ is the liquid density, $\mathrm{r}$ is the nozzle inner radius, $\nu$ is the liquid velocity, and $\gamma$ is the liquid surface tension) was chosen as an indicator ascertaining the system's operation in the simplejet mode. From the literature it is known that, for uncharged jets with $\mathrm{We} \geq 4$, the droplet formation mechanism happens in the jetting regime. ${ }^{26-33}$ Just below this value it happens in the so called transition regime, ${ }^{28}$ i.e., in between the dripping and the jetting regime. As mentioned by Cloupeau and Prunet-Foch ${ }^{23}$ for electrified jets this transition is expected to happen at lower Weber number values, corresponding to the transition regime, because the tangential component of the electric field increases liquid acceleration. We consider the simple-jet mode as the mode in which the breakup happens from an electrified jet but at flow rates in the order of the jetting regime and the transition regime.

The characteristics and definition of this mode has also been reported by Agostinho et al. ${ }^{34}$ According to the authors, it can be obtained when the liquid is electrosprayed at $W e \geq 4$ (jetting regime) or at lower values of $W e$ (transition regime) for certain values of the electric field. The spray size distribution was determined for three different flows $6.8 \times 10^{-8} \quad \mathrm{~m}^{3} \cdot \mathrm{s}^{-1} \quad(W e=3.3), \quad 1.02 \times 10^{-7} \quad \mathrm{~m}^{3} \cdot \mathrm{s}^{-1}$ $(W e=7.4)$, and $1.36 \times 10^{-7} \mathrm{~m}^{3} \cdot \mathrm{s}^{-1}(W e=13.4)$ for both liquids at different potentials (from 0 to $-10 \mathrm{kV}$ ). The lower flow $(W e=3.3)$ was chosen expressly to investigate whether the simple-jet mode could be imposed at $\mathrm{We} \leq 4$ and

TABLE I. Viscosity, density, relative permittivity, conductivity, and surface tension (liquid-air interface) of the liquids.

\begin{tabular}{lccccc}
\hline \hline Liquid & $\mu[\mathrm{Pa} \times \mathrm{s}]$ & $\rho\left[\mathrm{kg} \times \mathrm{m}^{3}\right]$ & $\varepsilon_{\mathrm{r}}$ & $\mathrm{K}\left[\mathrm{S} \times \mathrm{m}^{-1}\right]$ & $\gamma\left[\mathrm{N} \times \mathrm{m}^{-1}\right]$ \\
\hline Dwater & $1.00 \times 10^{-3}$ & $1.00 \times 10^{3}$ & $8.01 \times 10^{1}$ & $1.20 \times 10^{-3}$ & $7.19 \times 10^{-2}$ \\
NaCl aq. & $9.21 \times 10^{-4}$ & $1.05 \times 10^{3}$ & $7.35 \times 10^{1}$ & $4.5 \times 10^{0}$ & $7.3 \times 10^{-2}$ \\
\hline \hline
\end{tabular}

whether monodispersity could be achieved under these conditions.

The curves shown in Figure 2 are the particle size distribution of the electrosprayed droplets. They are plotted for two different values of We (3.3 and 13.4) at different potentials (from 0 to $10 \mathrm{kV}$ ) for the two tested liquids (Dwater and $\left.\mathrm{NaCl}_{\mathrm{aq}}\right)$.

From the curves, it is possible to see that inside the transition regime $(W e=3.3)$ the breakup at $\Phi=0$ creates a bimodal distribution (two peaks with similar frequencies). With the increase of the applied potential, the two peaks start to merge and a more monodisperse distribution is formed. We repeated this experiment for other values of the Weber number inside the transition regime ( $W e=3$ and 3.6) and the same effect was observed.

Inside the jetting regime $(W e \geq 4)$, the effect of the applied potential on droplet size distribution is noticeable but not strongly pronounced. As can be seen in Figures 2(b) and $2(\mathrm{~d})$, for $W e=13.4$ the breakup of the uncharged jet is already rather monodisperse. Longer critical wavelengths $\left(\lambda_{c}\right)$ are expected at this Weber number and, as a consequence, the formation of secondary or satellite droplets is likely. ${ }^{1,15,35}$ The presence of satellite droplets compromises the monodispersity because they cause a second class of diameters (smaller than the primary droplets) and they can coalesce with the main droplets generating a third (and bigger) class of diameters (tertiary droplets). This is seen in Figures 2(b) and 2(d) by the presence of the two extra peaks (left and right side of the primary droplet's peak). An effect of the electric potential is decreasing the third peak (tertiary droplets). This result is due to the electric charge acquired by the droplets during atomization which causes columbic repulsion and decreases coalescence effects.

In general, we noticed that an increase in potential causes a decrease of the satellite and primary droplet diameters. Regarding the primary droplets this happens because the electrostatic stresses decrease the jet radius, ${ }^{15,36,37}$ which shortens the wavelength of the fastest growing perturbation and consequently the droplet size. Similar phenomena were experimentally observed by Tang and Gomez for monodisperse sprays generated in the cone-jet mode. ${ }^{3,7}$ The influence of the applied potential on the satellite droplets has not been much explored in the literature. Some examples are the work of Collins et al. ${ }^{37}$ who have modelled the breakup of electrified jets and mentioned that the electrostatic stress increases the size of the satellite droplets; and the work of Hartman ${ }^{35}$ who reported that the presence and size of these droplets is a function of the electric current through the liquid jet in the cone-jet mode.

In the case of deionised water (Figure 2(b)), it was remarkable to observe that the peak associated with satellite droplets has almost disappeared for $\Phi=9 \mathrm{kV}$ and has returned at $\Phi=10 \mathrm{kV}$. This observation was reproducible and further investigations will be carried out to verify whether different electric fields (magnitude and direction) can be used to suppress the formation of satellite droplets and to change size distribution.

The broad size distribution presented for the two liquids for $W e=3.3$ at $\Phi=9 \mathrm{kV}$ (Figures 2(a) and 2(c)) and for the $\mathrm{NaCl}_{\mathrm{aq}}$ for $W e=13.4$ at $\Phi=10 \mathrm{kV}$ (Figure 2(d)) is a 

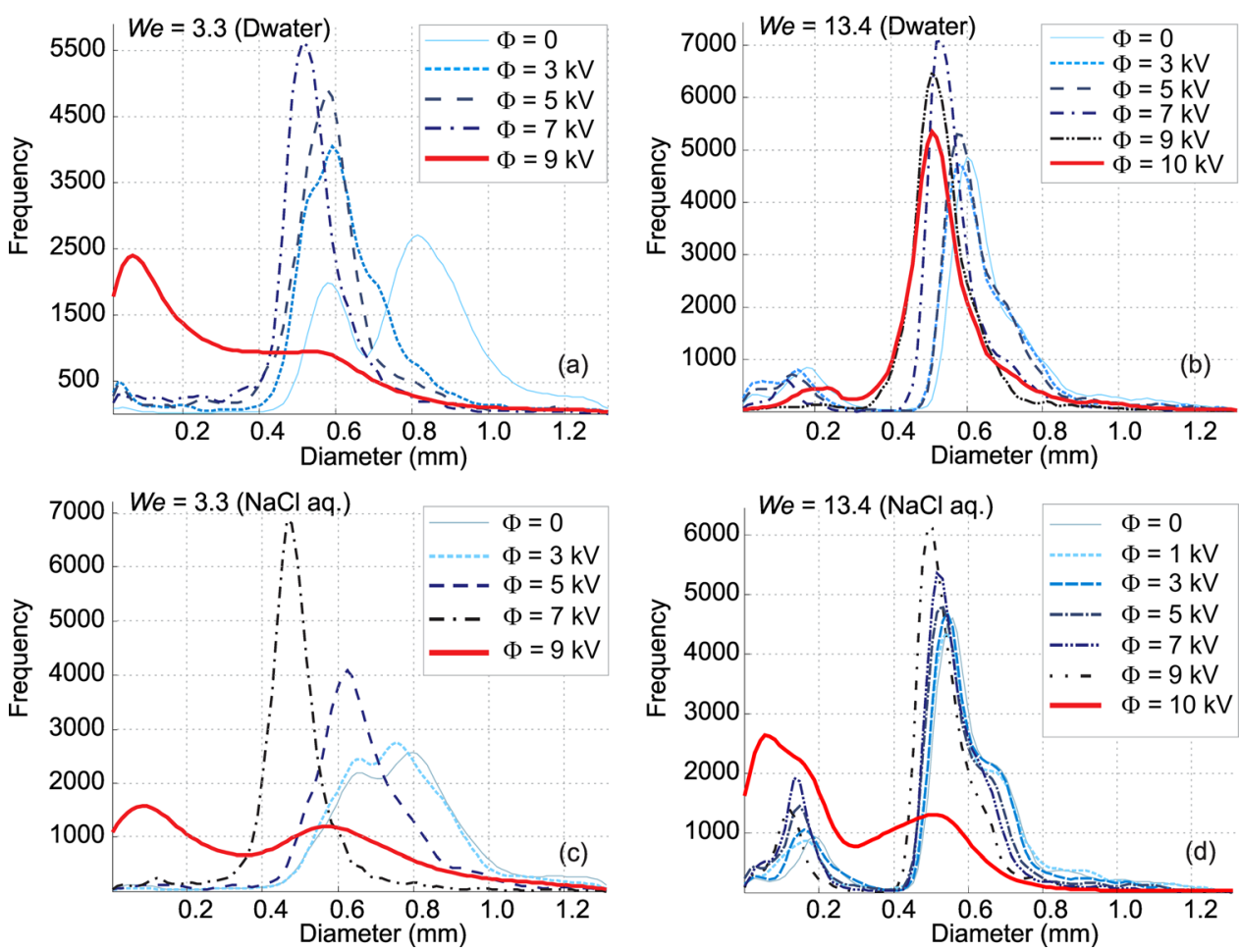

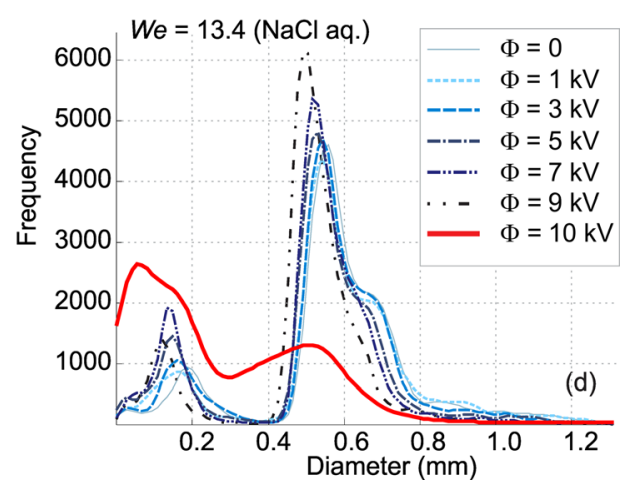

FIG. 2. (a)-(d) Particle size distribution of the droplet diameters for deionized water at $W e=3.3$ (a) and 13.4 (b) and $\mathrm{NaCl}$ aqueous solution at $W e=3.3$ (c) and $W e=13.4$ (d) for different potentials. consequence of whipping instabilities in the jet. As presented in literature, ${ }^{35}$ if the ratio between electrical and surface tension stresses overcomes a certain threshold, off axis instabilities develop in parallel to the axisymmetric instabilities making the size distribution broader. Figure 3 shows images of some sprays represented in Figure 2.

The bimodal distribution represented in Figures 2(a) and 2(c) can clearly be seen for $W e=3.3$ and $\Phi=0$. For the same value of $W e$, the figure also shows a good monodispersity at $\Phi=7 \mathrm{kV}$ and the whipping instabilities with a very broad size distribution for $\Phi=10 \mathrm{kV}$. For $W e=13.4$ the figure shows that the ligament between two main droplets decreases as the potential increases. Also, in the same sequence, the reduction on the primary droplet size for increasing potentials is noticeable especially for $W e=3.3$ (see also Figure 2(a)).

To quantitatively describe the influence of the applied potential on the droplets size distribution, we use the relative standard deviation (RSD). ${ }^{2,3,7}$

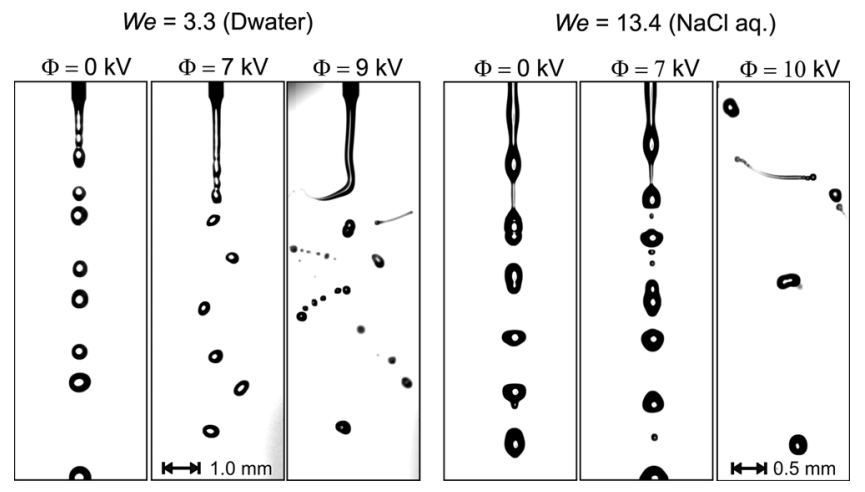

FIG. 3. Sprays at different potential and Weber number for Dwater and $\mathrm{NaCl}$.
Figure 4 shows that the RSD decreases with increasing potential for $W e=3.3$ and 13.4 for both liquids. The most significant reduction happens for $W e=3.3$ where the RSD decreases from 0.5 to 0.18 . Sprays with RSD smaller than 0.2 are considered monodisperse. ${ }^{7,38}$ For $W e=7.5$, the influence of the applied potential on the RSD is very small. This is due to the fact that such a spray is already rather monodisperse without the application of an electrical potential. Therefore, electrospraying the liquid under these conditions does not improve the droplet size distribution significantly. Comparing Figures 4(a) with 4(b) shows that the influence of the liquid's electrical conductivity on the RSD is not very pronounced as the values are similar for both liquids. Extra experiments (not shown) have indicated that higher conductivities cause whipping instabilities at slightly lower potentials, meaning that the potential range wherein a monodisperse distribution can be achieved decreases with increasing conductivity. RSDs above 0.6 indicate a whipping breakup.

For the specific kind of nozzle used in our experiments it was observed that, inside the jetting regime $(W e>4)$, for the uncharged condition $(\Phi=0)$, the diameter of the primary droplets is between 2.1 and 2.2 times bigger than the nozzle's inner diameter. The application of the electric potential reduces their diameter by approximately $20 \%$ at $\Phi=5 \mathrm{kV}$. In the transition regime $(W e=3.3)$, the reduction of the primary droplet size was up to $50 \%$ when compared to the uncharged situation. A similar result was found for the diameters of the satellite droplets which were also reduced up to $50 \%$.

These findings lead us to infer that the electric potential can significantly change the droplet size distribution in electrosprays operating in the simple-jet mode, especially for the transition regime, i.e., for $W e<4$. We have shown that for $W e=3.3$ the RSD of the distributions can be decreased from 

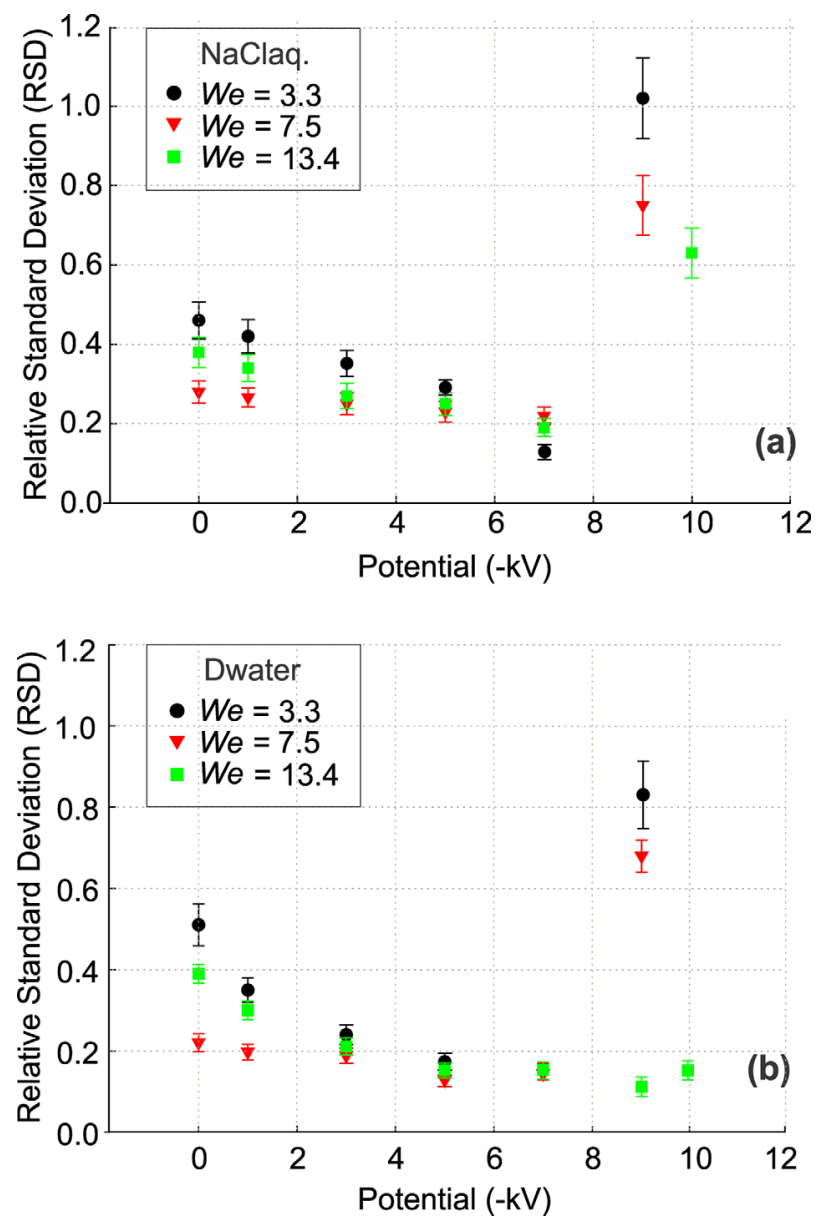

FIG. 4. RSD of the analyzed distributions for $\mathrm{NaCl}_{\mathrm{aq}}$ (a) and Dwater (b) for different values of $W e$ and $\Phi$.

0.5 in the uncharged situation to 0.18 at $5 \mathrm{kV}$, values comparable to those found for monodisperse electrosprays in the cone-jet mode. ${ }^{2,3,7}$ Sprays in the jetting regime already exhibited a good RSD, but the application of an electrical potential can significantly improve their monodispersity.

Simple-jet mode sprays are a very good option for applications which require monodispersity of droplets in the hundreds of micrometers range with much higher throughput as the flow required for the cone-jet mode.

This work was performed in the TTIW-cooperation framework of Wetsus, centre of excellence for sustainable water technology (www.wetsus.nl). Wetsus is funded by the Dutch Ministry of Economic Affairs. The authors would like to thank the participants of the research theme salt for the fruitful discussions and their financial support.

${ }^{1}$ N. Ashgriz, et al. Handbook of Atomization and Sprays (Springer, New York, NY, 2011).
${ }^{2}$ K. Tang and A. Gomez, J. Colloid Interface Sci. 175(2), 326-332 (1995). ${ }^{3}$ K. Tang and A. Gomez, J. Colloid Interface Sci. 184(2), 500-511 (1996).

${ }^{4}$ I. G. Loscertales, A. Barrero, I. Guerrero, R. Cortijo, M. Marquez, and A. M. Ganan-Calvo, Science 295(5560), 1695-1698 (2002).

${ }^{5}$ L. Gradon and J. Marijnissen, Optimization of Aerosol Drug Delivery (Kluwer Academic, Dordrecht, 2003).

${ }^{6}$ W. Deng, C. M. Waits, B. Morgan, and A. Gomez, J. Aerosol Sci. 40(10), 907-918 (2009).

${ }^{7}$ K. Tang and A. Gomez, J. Aerosol Sci. 25(6), 1237-1249 (1994).

${ }^{8}$ D. B. Bogy, Annu. Rev. Fluid Mech. 11, 207-228 (1979).

${ }^{9}$ D. M. Mitchell, M. A. Solomon, S. E. Tolfree, M. Short, and S. G. Spiro, Thorax 42(6), 457-461 (1987).

${ }^{10}$ G. M. H. Meesters, P. H. W. Vercoulen, J. C. M. Marijnissen, and B. Scarlett, J. Aerosol Sci. 21 (Supplement 1(0)), S669-S672 (1990).

${ }^{11}$ F. A. Williams, Phys. Fluids 1(6), 541-545 (1958).

${ }^{12}$ A. M. Gañán-Calvo, Phys. Rev. Lett. 80(2), 285-288 (1998).

${ }^{13}$ H. Liu, Science and Egineering of Droplets (William Andrew, New York, 1981).

${ }^{14}$ L. Rayleigh, Proc. R. Soc. London 29, 71-97 (1879).

${ }^{15}$ R. P. A. Hartman, D. J. Brunner, D. M. A. Camelot, J. C. M. Marijnissen, and B. Scarlett, J. Aerosol Sci. 31(1), 65-95 (2000).

${ }^{16}$ L. L. F. Agostinho, J. Marijnissen, C. U. Yurteri, E. Fuchs, and S. Metz, Phys. Rev. E 84, 026317 (2011).

${ }^{17}$ A. M. Ganan-Calvo and J. M. Montanero, Phys. Rev. E 79(6), 066305 (2009).

${ }^{18}$ M. A. Herrada and A. M. Gañán-Calvo, Phys. Fluids 21(4), 042003 (2009).

${ }^{19}$ Y. Arnanthigo, J. Marijnissen, C. U. Yurteri, G. Biskos, and A. SchmidtOtt, Powder Technol. 214, 382(6) (2011).

${ }^{20}$ M. Cloupeau and B. Prunet-Foch, J. Electrost. 22(2), 135-159 (1989).

${ }^{21}$ D. M. A. Camelot, D. Brunner, R. P. M. Hartman, J. C. M. Marijnissen, and B. Scarlett, J. Aerosol Sci. 29(Supplement 2), S841S842 (1998).

${ }^{22}$ R. Hartman, Electrohydrodynamic Atomization in the Cone-Jet Mode From Physical Modeling to Powder Production (Delft University of Technology, 1998).

${ }^{23}$ M. Cloupeau and B. Prunet-Foch, J. Aerosol Sci. 25(6), 1021-1036 (1994).

${ }^{24}$ J. M. Grace and J. C. M. Marijnissen, J. Aerosol Sci. 25(6), 1005-1019 (1994).

${ }^{25}$ K. B. Geerse, Applications of Electrospray: from people to plants (Delft University of Technology, 2003).

${ }^{26}$ B. Ambravaneswaran, S. D. Phillips, and O. A. Basaran, Phys. Rev. Lett. 85(25), 5332-5335 (2000).

${ }^{27}$ B. Ambravaneswaran, H. J. Subramani, S. D. Phillips, and O. A. Basaran, Phys. Rev. Lett. 93(3), 034501 (2004).

${ }^{28}$ C. Clanet and J. C. Lasheras, J. Fluid Mech. 383, 307-326 (1999).

${ }^{29}$ W. V. Hoeve, S. Gekle, J. H. Snoeijer, M. Versluis, M. P. Brenner, and D. Lohse, Phys. Fluids 22(12), 122003 (2010).

${ }^{30}$ P. Lafrance, Phys. Fluids 18(4), 428-432 (1975).

${ }^{31}$ J. Eggers and E. Villermaux, Rep. Prog. Phys. 71(3), 036601 (2008).

${ }^{32}$ S. P. Lin, Breakup of Liquid Sheets and Jets (Cambridge University Press, 2010).

${ }^{33}$ M. Rohani, F. Jabbari, and D. Dunn-Rankin, Physics of Fluids 22(10), 107103 (2010).

${ }^{34}$ L. L. F. Agostinho, J. Wartena, E. C. Fuchs, C. U. Yurteri, and J. C. M. Marijnissen, "Characterization of EHDA in the simple-jet mode," presented at the European Aerosol Conference, Manchester, UK (unpublished).

${ }^{35}$ R. P. A. Hartman, D. J. Brunner, D. M. A. Camelot, J. C. M. Marijnissen, and B. Scarlett, J. Aerosol Sci. 30(7), 823-849 (1999).

${ }^{36}$ A. L. Huebner and H. N. Chu, J. Fluid Mech. 49(02), 361-372 (1971).

${ }^{37}$ R. T. Collins, M. T. Harris, and O. A. Basaran, J. Fluid Mech. 588, 75-129 (2007).

${ }^{38}$ P. A. Baron, K. Willeke, and P. Kulkarni, Aerosol Measurements: Principle, Techniques and Applications, 3rd ed. (Wiley, 2011). 